\title{
ANDES

www.scielo.cl

\section{Respuesta carta al editor titulada: Variación en los parámetros predictores de gravedad en el Síndrome Inflamatorio Pediátrico Multisistémico asociado a SARS-CoV 2}

\section{Response to the letter of editor entitled: Discrepencies in Severity Predictive Parameters in Multisystemic Pediatric Inflammatory Syndrome Associated with SARS-CoV 2}

\section{Patricia Verdugo ${ }^{\mathrm{a}, \mathrm{b}}$}

aServicio de Onco-Hematología. Hospital Roberto del Río. Santiago, Chile

bDepartamento de Pediatría, Campus Norte, Facultad de Medicina, Universidad de Chile. Santiago, Chile

\section{Sr. Editor,}

Me parece interesante la comparación de nuestro trabajo de parámetros hematológicos y biomarcadores predictores de gravedad en MIS-C con otras publicaciones extranjeras, sin embargo, quiero destacar que nuestra revisión se hizo en 32 pacientes pediátricos y no 21 como muestra la tabla comparativa. Quiero señalar que en las referencias bibliográficas mencionadas engloban a pacientes pediátricos con infección aguda y MIS-C con lo cual podrían haber diferencias en los resultados de laboratorios estadísticamente significativo como es la linfopenia. Me parece muy interesante investigar factores de riesgo de gravedad clínico como la obesidad o comorbilidad asociada, hemos observado que la obesidad en los niños con infección aguda por COVID 19 aumenta la gravedad de la evolución clínica, similar a los adultos .

Creo que es de gran aporte en mejorar el conocimiento de esta nueva y grave patología en la población pediátrica ayudando de esta forma al clínico en su manejo. 